\title{
CONTINUOUS DEPENDENCE RESULTS FOR A PROBLEM IN PENETRATIVE CONVECTION
}

\author{
BY \\ KAREN A. AMES (Department of Mathematical Sciences, University of Alabama in Huntsville) \\ AND \\ LAWRENCE E. PAYNE (Department of Mathematics, Cornell University, Ithaca, New York)
}

\begin{abstract}
Continuous dependence inequalities are derived for a system of equations that models penetrative convection in a thermally conducting viscous fluid with a linear buoyancy law. Both the forward-in-time problem and the improperly posed backwardin-time problem are analyzed. These results indicate that solutions depend continuously on a parameter in the boundary data.
\end{abstract}

1. Introduction. The phenomenon of penetrative convection has attracted the attention of a number of investigators in diverse fields. Various models have been formulated to describe this process. A recent monograph by Straughan [11] reviews some of the mathematical aspects of these models, with an emphasis on the derivation of continuous dependence results for solutions of properly and improperly posed initial-boundary value problems. Most of these convection models consist of coupled systems of partial differential equations that include the Navier-Stokes equations. Consequently, a study of penetrative convection often rests on an analysis of the Navier-Stokes system. A number of investigations on the question of continuous dependence of solutions to this system on various types of data, both forward and backward in time, have appeared recently in the literature. These include the study of Franchi and Straughan [4] who establish continuous dependence on the body force for solutions of the system backward in time, the work of Song [9] on well-posed problems, and a paper by Ames and Payne [2] that deals with stabilizing the Navier-Stokes system backward in time against errors in the body force, coefficient of kinematic viscosity, and boundary data. The earliest study of the Navier-Stokes system backward in time has been attributed to Serrin [8] who established uniqueness of the solution to the equations defined on a bounded spatial domain. The task of stabilizing the final value problem against errors in the final data was first addressed by Knops and Payne [5]. In this paper, we continue to investigate the NavierStokes system but couple it with a convective heat equation. The resulting system models

Received February 24, 1995.

1991 Mathematics Subject Classification. Primary 35K60, 35Q30, 35R25, 76D05, 35B30.

This research was supported in part by NSF grant DMS-9308121. 
penetrative convection in a thermally conducting viscous fluid with a linear buoyancy law.

Our goal then is to derive inequalities indicating that a solution to the Navier-Stokes equations coupled with the convective heat equation depends continuously on a parameter specified in the boundary data. We shall consider both the forward-in-time problem and the improperly posed backward-in-time problem on bounded spatial domains. Let $\Omega \subseteq \mathbb{R}^{n}(n=2,3)$ be such a domain with boundary $\Gamma$. If $\Omega \subset \mathbb{R}^{2}$, then we require $\Gamma$ to be Lipschitz while for domains in $\mathbb{R}^{3}$, we assume $\Gamma$ is a $C^{2}$ surface. We consider the system

$$
\begin{aligned}
v_{i . t}+v_{j} v_{i, j} & =-p,_{i}+\Delta v_{i}+b_{i} T, \\
v_{i, i} & =0, \\
T,_{t}+v_{i} T,_{i} & =\Delta T
\end{aligned}
$$

defined on $\Omega \times\left(0, t_{0}\right)$. The auxiliary conditions we associate with these equations take the form of the boundary conditions

$$
\left.\begin{array}{r}
v_{i}=0 \\
T,{ }_{i} n_{i}+\kappa T=0
\end{array}\right\} \quad \text { on } \Gamma \times\left[0, t_{0}\right]
$$

and the initial conditions

$$
\left.\begin{array}{l}
v_{i}(x, 0)=v_{i}^{0}(x) \\
T(x, 0)=T_{0}(x)
\end{array}\right\} \quad \text { for } x \in \Omega .
$$

Here $v_{i}(x, t)$ is the $i$ th $(i=1,2,3)$ component of the fluid velocity, $p$ is the modified pressure, $T(x, t)$ is the temperature, and $b_{i}(x, t)$ is the body force. Without loss of generality, we have assumed that the coefficient of viscosity and thermal diffusivity are unity. Standard indicial notation is used throughout this paper and differentiation is denoted by a comma. We shall restrict our attention to classical solutions of (1.1)-(1.5), which we assume to exist on the time interval $\left(0, t_{0}\right)$. In addition, we assume, for the problem in $\mathbb{R}^{3}$, that the data are sufficiently smooth and compatible so that the partial differential equation (1.3) is satisfied on $t=0$.

In the next section we derive some supplementary inequalities that we need to establish our main results. Sections 3 and 4 are devoted to the forward-in-time problem defined for $\Omega \subseteq \mathbb{R}^{3}$ and $\Omega \subseteq \mathbb{R}^{2}$, respectively. We turn to the ill-posed backward-in-time problem in Sec. 5. Some of the bounds we need to establish continuous dependence on the boundary parameter for solutions of the forward-in-time problem are included in the appendix.

As is to be expected, the results for the forward problem in $\mathbb{R}^{3}$ are much less satisfactory than those in $\mathbb{R}^{2}$. To derive the continuous dependence result in $\mathbb{R}^{3}$ we require more smoothness and compatibility, and some restriction on the size of the data is required if the result is to hold on $\left(0, t_{0}\right)$. For the ill-posed backward-in-time problem, solutions must of course be more severely constrained.

2. Useful inequalities. In the subsequent sections we will make frequent use of an assortment of integral inequalities. We list here a number of those which will be needed in the later sections. 
We remark first that on the interval $\left(0, t_{0}\right)$ where $v_{i}$ is assumed to be bounded, $T$ satisfies the maximum principle for parabolic equations, and it follows that

$$
|T| \leq \max _{\Omega}\left|T_{0}\right|
$$

The maximum value of $|T|$ cannot occur on $\partial \Omega$ for $0 \leq t \leq t_{0}$, since if it did occur at a point $P$ on $\partial \Omega$ at time $\hat{t}$ then if $T(P, \hat{t})>0$ it follows that $\frac{\partial T}{\partial n}(P, \hat{t})<0$, a contradiction. Similarly if $T(P, \hat{t})<0$ then $\frac{\partial T}{\partial n}(P, \hat{t})>0$, again a contradiction. Thus, the maximum value of $|T|$ must occur on $t=0$.

We now derive two $L_{p}$ inequalities for $T$. The second of these inequalities will be used with various values of $p$ in our proofs in Secs. 3 and 4 . We show, in particular, that

$$
\int_{\Omega} T^{2 p} d x+2 p \kappa \int_{0}^{t} \int_{\Gamma} T^{2 p} d \sigma d \eta+2 p(2 p-1) \int_{0}^{t} \int_{\Omega} T^{2 p-2} T,_{i} T,_{i} d x d \eta=\int_{\Omega} T_{0}^{2 p} d x
$$

and

$$
\int_{\Omega} T^{2 p} d x \leq\left[\int_{\Omega} T_{0}^{2 p} d x\right] \exp \left\{-2 p^{-1}(2 p-1) \mu(\kappa, p) t\right\}
$$

where

$$
\mu(\kappa, p)=\inf _{\varphi \in \mathcal{D}} \frac{\int_{\Omega} \varphi,_{i} \varphi_{i} d x+\frac{p^{2} \kappa}{2 p-1} \int_{\Gamma} \varphi^{2} d \sigma}{\int_{\Omega} \varphi^{2} d x},
$$

and $\mathcal{D}$ is the class of Dirichlet integrable functions having $L_{2}$ boundary integrals. Bounds for $\mu(k, p)$ have been given by Payne and Weinberger [7], Sperb [10], etc.

The inequalities (2.2) and (2.3) follow from the observation that

$$
\frac{d}{d t} \int_{\Omega} T^{2 p} d x=-2 p \kappa \int_{\Gamma} T^{2 p} d \sigma-2 p(2 p-1) \int_{\Omega} T^{2 p-2} T,_{i} T,_{i} d x .
$$

We have used the differential equation for $T$ and (1.2). An integration of (2.5) leads to (2.2) while the rewriting of (2.5) as

$$
\frac{d}{d t} \int_{\Omega} T^{2 p} d x=-2 p \kappa \int_{\Gamma}\left[T^{2}\right]^{p} d \sigma-\frac{2(2 p-1)}{p} \int_{\Omega}\left(T^{p}\right)_{, i}\left(T^{p}\right),_{i} d x
$$

and use of (2.4) leads to

$$
\frac{d}{d t} \int_{\Omega} T^{2 p} d x \leq-2 p^{-1}(2 p-1) \mu(\kappa, p) \int_{\Omega} T^{2 p} d x .
$$

An integration of (2.7) then yields (2.3).

We next derive a differential inequality for the $L_{2}$ integral of the velocity. Proceeding directly we have

$$
\begin{aligned}
\frac{d}{d t}\left[\int_{\Omega} v_{i} v_{i} d x\right] & =2 \int_{\Omega} v_{i}\left\{-p_{,}+\Delta v_{i}+b_{i} T-v_{j} v_{i},{ }_{j}\right\} d x \\
& =-2 \int_{\Omega} v_{i, j} v_{i, j} d x+2 \int_{\Omega} b_{i} v_{i} T d x \\
& \leq-2 \int_{\Omega} v_{i, j} v_{i, j} d x+2\left[b_{i} b_{i}\right]_{\max }^{1 / 2}\left\{\int_{\Omega} v_{i} v_{i} d x \int_{\Omega} T^{2} d x\right\}^{1 / 2}
\end{aligned}
$$


By rescaling $T$ if necessary we may assume that

$$
b_{i} b_{i}<1
$$

Thus for any positive constant $\gamma_{1}$ it follows that

$$
\begin{aligned}
\frac{d}{d t}\left[\int_{\Omega} v_{i} v_{i} d x\right] & \leq-2 \int_{\Omega} v_{i, j} v_{i, j} d x+\gamma_{1} \int_{\Omega} v_{i} v_{i} d x+\gamma_{1}^{-1} \int_{\Omega} T^{2} d x \\
& \leq-\left(2 \lambda-\gamma_{1}\right) \int_{\Omega} v_{i} v_{i} d x+\gamma_{1}^{-1}\left[\int_{\Omega} T_{0}^{2} d x\right] \exp \{-\mu(\kappa, 1) t\}
\end{aligned}
$$

We have used (2.2) with $p=1$ and introduced the constant $\lambda$ which is defined as

$$
\lambda=\inf \frac{\int_{\Omega} \psi_{i, j} \psi_{i, j} d x}{\int_{\Omega} \psi_{i} \psi_{i} d x}
$$

where $\psi_{i}$ lies in the class of divergence-free piecewise continuously differentiable functions that vanish on $\Gamma$. Velte [12] has shown that in $\mathbb{R}^{2}, \lambda$ is precisely the first eigenvalue in the corresponding plate buckling problem. In higher dimensions $\lambda$ remains larger than the corresponding first eigenvalue of the Laplacian. So in the next section we use this first eigenvalue of the Laplacian, the so-called first clamped membrane eigenvalue, for our constant $\lambda$.

The inequality $(2.10)$ is of the form

$$
\frac{d \Psi}{d t} \leq-k_{1} \Psi+k_{2} e^{-\mu(\kappa .1) t}
$$

which integrates to give (suppressing the arguments of $\mu$ )

$$
\Psi(t) \leq \Psi(0) e^{-k_{1} t}+\frac{k_{2}}{k_{1}-\mu}\left[e^{-\mu t}-e^{-k_{1} t}\right]
$$

or

$$
\int_{\Omega} v_{i} v_{i} d x \leq \int_{\Omega} v_{i}^{0} v_{i}^{0} d x e^{-\left(2 \lambda-\gamma_{1}\right) t}+\frac{\left(\int_{\Omega} T_{0}^{2} d x\right)}{\gamma_{1}\left[2 \lambda-\gamma_{1}-\mu\right]}\left[e^{-\mu t}-e^{-\left(2 \lambda-\gamma_{1}\right) t}\right] .
$$

Choosing

$$
\gamma_{1}=2 \lambda-\mu(\kappa, 1)
$$

we are led to the decay bound

$$
\int_{\Omega} v_{i} v_{i} d x \leq \int_{\Omega} v_{i}^{0} v_{i}^{0} d x e^{-\mu(\kappa, 1) t}+\frac{t}{[2 \lambda-\mu(\kappa, 1)]} \int_{\Omega} T_{0}^{2} d x e^{-\mu(\kappa, 1) t} .
$$

Returning to (2.8) we observe that

$$
\begin{aligned}
\frac{d}{d t}\left[\int_{\Omega} v_{i} v_{i} d x\right]= & -2 \int_{\Omega} v_{i, j} v_{i, j} d x+2\left\{\int_{\Omega} T_{0}^{2} d x e^{-\mu(\kappa .1) t}\right\}^{1 / 2} \\
& \times\left\{\int_{\Omega} v_{i}^{0} v_{i}^{0} d x e^{-\mu(\kappa, 1) t}+\frac{t}{[2 \lambda-\mu(\kappa, 1)]} \int_{\Omega} T_{0}^{2} d x e^{-\mu(\kappa, 1) t}\right\}^{1 / 2}
\end{aligned}
$$


or upon integration

$$
\begin{aligned}
& 2 \int_{0}^{t} \int_{\Omega} v_{i, j} v_{i, j} d x d \eta+\int_{\Omega} v_{i} v_{i} d x \\
& \quad \leq \int_{\Omega} v_{i}^{0} v_{i}^{0} d x+K_{1} \int_{0}^{t} \eta e^{-\mu(\kappa, 1) \eta} d \eta+K_{2} \int_{0}^{t} e^{-\mu(\kappa, 1) \eta} d \eta
\end{aligned}
$$

for computable $K_{1}$ and $K_{2}$. It follows then that for a computable $K$ (which depends on the data but is independent of $t_{0}$ )

$$
2 \int_{0}^{t} \int_{\Omega} v_{i, j} v_{i, j} d x d \eta+\int_{\Omega} v_{i} v_{i} d x \leq K .
$$

In the next two sections we also require a bound for the quantity $\int_{0}^{t} \int_{\Gamma} e^{-\beta(t-\eta)} T^{2} d \sigma d \eta$ for specific values of the positive constant $\beta$. To find such a bound we first note that

$$
\frac{1}{2} \int_{\Gamma} T^{2} d \sigma+\int_{\Omega} T,_{i} T,_{i} d x=-\int_{\Omega} T \Delta T d x=-\int_{\Omega} T T,_{t} d x
$$

from which we have

$$
\begin{aligned}
\int_{0}^{t} \int_{\Gamma} e^{-\beta(t-\eta)} T^{2} d \sigma d \eta & \leq-2 \int_{0}^{t} \int_{\Omega} e^{-\beta(t-\eta)} T T,_{\eta} d x d \eta \\
& \leq\left[\int_{\Omega} T_{0}^{2} d x\right] e^{-\beta t}+\beta \int_{0}^{t} \int_{\Omega} e^{-\beta(t-\eta)} T^{2} d x d \eta
\end{aligned}
$$

Using (2.3) in the last term we obtain

$$
\int_{0}^{t} \int_{\Gamma} e^{-\beta(t-\eta)} T^{2} d \sigma d \eta \leq \frac{2 \mu(\kappa, 1)}{[2 \mu(\kappa, 1)-\beta]}\left[\int_{\Omega} T_{0}^{2} d x\right] e^{-\beta t}
$$

the bound that we will be using in the next two sections.

Finally, we list two Sobolev inequalities which will prove useful. For Dirichlet integrable functions, $\varphi$, defined on $\Omega$ and vanishing on $\Gamma$ we have

$$
\int_{\Omega} \varphi^{4} d x \leq \Lambda^{2}\left(\int_{\Omega} \varphi^{2} d x\right)\left(\int_{\Omega} \varphi,{ }_{i} \varphi,,_{i} d x\right) \text { in } \mathbb{R}^{2}
$$

and

$$
\int_{\Omega} \varphi^{4} d x \leq \nu^{2}\left(\int_{\Omega} \varphi^{2} d x\right)^{1 / 2}\left(\int_{\Omega} \varphi,{ }_{i} \varphi,{ }_{i} d x\right)^{3 / 2} \text { in } \mathbb{R}^{3} .
$$

For specific values of $\Lambda$ and $\nu$, see [1]. It has been known for some time that $\Lambda \leq 2^{-1 / 2}$ and $\nu \leq 3^{-3 / 4}$, [8].

Bounds for various other norms of $v_{i}$ and $T$ will be derived in the Appendix.

3. The forward-in-time problem in $\mathbb{R}^{3}$. Let $\left(v_{i}^{*}, T^{*}\right)$ be the solution of $(1.1)-(1.3)$ with $p=p^{*}$ and satisfying

$$
\left.\begin{array}{r}
v_{i}^{*}=0 \\
T,{ }_{i}^{*} n_{i}+\kappa^{*} T^{*}=0
\end{array}\right\} \quad \text { on } \Gamma \times\left[0, t_{0}\right]
$$


as well as

$$
\left.\begin{array}{r}
v_{i}^{*}(x, 0)=v_{i}^{0}(x) \\
T^{*}(x, 0)=T_{0}(x)
\end{array}\right\} \quad \text { for } x \in \Omega .
$$

Our goal is to show that if $\kappa^{*}$ is close to $\kappa$ then the perturbation $\left(u_{i}, \theta\right)$ defined as

$$
\begin{aligned}
u_{i} & =v_{i}^{*}-v_{i}, \\
\theta & =T^{*}-T
\end{aligned}
$$

will be small in some appropriate measure. Specifically, we derive an inequality of the form

$$
\int_{\Omega}\left[a u_{i} u_{i}+\theta^{2}\right] d x \leq\left(\kappa^{*}-\kappa\right)^{2} F(t)
$$

for some positive constant $a$ and a function $F(t)$.

We first note that $u_{i}$ and $\theta$ satisfy

$$
\begin{aligned}
& u_{i, t}+v_{j}^{*} u_{i, j}+u_{j} v_{i, j}=-P,_{i}+\Delta u_{i}+b_{i} \theta \\
& u_{i, i}=0 \quad \text { in } \Omega \times\left(0, t_{0}\right) \\
& \theta,{ }_{t}+v_{i}^{*} \theta,_{i}+u_{i} T,_{i}=\Delta \theta
\end{aligned}
$$

with

$$
\left.\begin{array}{c}
u_{i}=0 \\
\theta,{ }_{i} n_{i}+\kappa \theta=-\alpha T^{*}
\end{array}\right\} \quad \text { on } \Gamma \times\left[0, t_{0}\right]
$$

and

$$
\theta(x, 0)=u_{i}(x, 0)=0, \quad x \in \Omega
$$

Here $P=p^{*}-p$ and

$$
\alpha=\kappa^{*}-\kappa .
$$

For some positive constant $a$, to be determined later, we now define the function $\Phi(t)$ given by

$$
\Phi(t)=\int_{\Omega}\left[\theta^{2}+a u_{i} u_{i}\right] d x
$$

We will derive a first-order differential inequality for $\Phi(t)$ which will yield a result of the type (3.4).

Differentiating (3.11) we have

$$
\begin{aligned}
\frac{d \Phi}{d t}= & 2 \int_{\Omega}\left[\theta\left\{\Delta \theta-v_{i}^{*} \theta,_{i}-u_{i} T,_{i}\right\}+a u_{i}\left\{-P,{ }_{i}+\Delta u_{i}+b_{i} \theta-v_{j}^{*} u_{i, j}-u_{j} v_{i, j}\right\}\right] d x \\
= & -2 \int_{\Omega} \theta,_{i} \theta,_{i} d x-2 \kappa \int_{\Gamma} \theta^{2} d \sigma-2 \alpha \int_{\Gamma} T^{*} \theta d \sigma+2 \int_{\Omega} u_{i} \theta,_{i} T d x \\
& -2 a\left\{\int_{\Omega} u_{i, j} u_{i, j} d x+\int_{\Omega} u_{i} u_{j} v_{i},{ }_{j} d x-\int_{\Omega} b_{i} \theta u_{i} d x\right\} .
\end{aligned}
$$


We have used (3.5)-(3.10). Let us consider some of the terms separately. For instance, from the Schwarz and Hölder inequalities and (2.24) we have

$$
\begin{aligned}
\left|\int_{\Omega} u_{i} \theta,_{i} T d x\right| & \leq\left[\int_{\Omega} \theta,,_{i} \theta,,_{i} d x\right]^{1 / 2}\left[\int_{\Omega} u_{i} u_{i} T^{2} d x\right]^{1 / 2} \\
& \leq\left[\int_{\Omega} \theta,_{i} \theta,,_{i} d x\right]^{1 / 2}\left[\int_{\Omega}\left|u_{i} u_{i}\right|^{3 / 2} d x\right]^{1 / 3}\left[\int_{\Omega} T^{6} d x\right]^{1 / 6} \\
& \leq\left[\int_{\Omega} \theta,,_{i} \theta,,_{i} d x\right]^{1 / 2}\left[\int_{\Omega} u_{i} u_{i} d x \int_{\Omega}\left[u_{i} u_{i}\right]^{2} d x\right]^{1 / 6}\left[\int_{\Omega} T^{6} d x\right]^{1 / 6} \\
& \leq \nu^{1 / 3}\left[\int_{\Omega} \theta,,_{i} \theta,,_{i} d x\right]^{1 / 2}\left[\int_{\Omega} u_{i} u_{i} d x \int_{\Omega} u_{i, j} u_{i}, j_{j} d x\right]^{1 / 4}\left[\int_{\Omega} T^{6} d x\right]^{1 / 6} .
\end{aligned}
$$

Thus

$$
\begin{aligned}
& \left|\int_{\Omega} u_{i} \theta,,_{i} T d x\right| \\
& \leq \nu^{1 / 3}\left[\int_{\Omega} \theta,_{i} \theta,_{i} d x\right]^{1 / 2}\left[\int_{\Omega} u_{i} u_{i} d x \int_{\Omega} u_{i},{ }_{j} u_{i},{ }_{j} d x\left\{\int_{\Omega} T^{6} d x\right\}^{2 / 3}\right]^{1 / 4} \\
& \leq \nu^{1 / 3} \frac{\gamma_{1}^{-1}}{2} \int_{\Omega} \theta,_{i} \theta,_{i} d x+\frac{\nu^{1 / 3} \gamma_{1}}{2}\left[\int_{\Omega} u_{i} u_{i} d x \int_{\Omega} u_{i, j} u_{i},{ }_{j} d x\left(\int_{\Omega} T^{6} d x\right)^{2 / 3}\right]^{1 / 2} \\
& \leq \nu^{1 / 3} \frac{\gamma_{1}^{-1}}{2} \int_{\Omega} \theta,{ }_{i} \theta,,_{i} d x+\nu^{1 / 3} \frac{\gamma_{1} \gamma_{2}}{4} \int_{\Omega} u_{i, j} u_{i, j} d x \\
& \quad+\frac{\nu^{1 / 3} \gamma_{1} \gamma_{2}^{-1}}{4}\left(\int_{\Omega} T^{6} d x\right)^{2 / 3} \int_{\Omega} u_{i} u_{i} d x
\end{aligned}
$$

for arbitrary positive constants $\gamma_{1}$ and $\gamma_{2}$. We will use inequality (2.3) to bound $\int_{\Omega} T^{6} d x$, but first let us bound other terms in (3.12). Clearly

$$
\begin{aligned}
\left|\int_{\Omega} u_{i} u_{j} v_{i, j} d x\right| & \leq\left[\int_{\Omega}\left[u_{i} u_{i}\right]^{2} d x \int_{\Omega} v_{k, j} v_{k, j} d x\right]^{1 / 2} \\
& \leq \nu\left[\int_{\Omega} u_{i} u_{i} d x\right]^{1 / 4}\left[\int_{\Omega} u_{k, j} u_{k, j} d x\right]^{3 / 4}\left[\int_{\Omega} v_{l, m} v_{l, m} d x\right]^{1 / 2} \\
& \leq \frac{\nu}{4}\left\{\gamma_{3}^{3} \int_{\Omega} u_{i} u_{i} d x\left[\int_{\Omega} v_{k, j} v_{k, j} d x\right]^{2}+3 \gamma_{3}^{-1} \int_{\Omega} u_{i, j} u_{i, j} d x\right\}
\end{aligned}
$$

for arbitrary positive $\gamma_{3}$. We have used Young's inequality in the last step. We note also that

$$
\left|\alpha \int_{\Gamma} T^{*} \theta d \sigma\right| \leq \frac{\alpha^{2}}{2} \gamma_{4} \int_{\Gamma}\left[T^{*}\right]^{2} d \sigma+\frac{\gamma_{4}^{-1}}{2} \int_{\Gamma} \theta^{2} d \sigma
$$


and

$$
\left|\int_{\Omega} b_{i} \theta u_{i} d x\right| \leq \frac{\gamma_{5}}{2} \int_{\Omega} \theta^{2} d x+\frac{\gamma_{5}^{-1}}{2} \int_{\Gamma} u_{i} u_{i} d x
$$

where we have made use of (2.9).

We now insert (3.14)-(3.17) into (3.12) to obtain

$$
\begin{aligned}
\frac{d \Phi}{d t} \leq & -\left(2-\nu^{1 / 3} \gamma_{1}^{-1}\right) \int_{\Omega} \theta,_{i} \theta,_{i} d x-\left(2 \kappa-\gamma_{4}^{-1}\right) \int_{\Gamma} \theta^{2} d \sigma+a \gamma_{5} \int_{\Omega} \theta^{2} d x \\
& +\alpha^{2} \gamma_{4} \int_{\Gamma}\left[T^{*}\right]^{2} d \sigma \\
& -\left(2 a-\frac{\nu^{1 / 3} \gamma_{1} \gamma_{2}}{2}-\frac{3 \nu \gamma_{3}^{-1} a}{2}\right) \int_{\Omega} u_{i, j} u_{i, j} d x \\
& +\left\{\gamma_{5}^{-1} a+\frac{\nu \gamma_{3}^{3}}{2} a\left[\int_{\Omega} v_{i, j} v_{i, j} d x\right]^{2}+\frac{\nu^{1 / 3}}{2} \gamma_{1} \gamma_{2}^{-1} \int_{\Omega \Omega}\left[T^{6} d x\right]^{2 / 3}\right\} \int_{\Omega} u_{i} u_{i} d x .
\end{aligned}
$$

If we make the specific choices

$$
\gamma_{1}=\nu^{1 / 3} ; \quad \gamma_{2}=a \nu^{-2 / 3} ; \quad \gamma_{3}=3 \mu ; \quad \gamma_{4}=\kappa^{-1}
$$

and make use of (2.4) and (2.11) we find

$$
\begin{aligned}
\frac{d \Phi}{d t} \leq- & {\left[\mu(\kappa, 1)-a \gamma_{5}\right] \int_{\Omega 2} \theta^{2} d x+\alpha^{2} \kappa^{-1} \int_{\Gamma}\left[T^{*}\right]^{2} d \sigma-a\left[\lambda-\gamma_{5}^{-1}\right] \int_{\Omega \Omega} u_{i} u_{i} d x } \\
+ & \frac{27 \nu^{4} a}{2}\left[\int_{\Omega} v_{i . j} v_{i . j} d x\right]^{2} \int_{\Omega \Omega} u_{i} u_{i} d x+\frac{\nu^{4 / 3}}{2 a}\left[\int_{\Omega} T^{6} d x\right]^{3 / 2} \int_{\Omega} u_{i} u_{i} d x .
\end{aligned}
$$

We next choose

$$
\gamma_{5}=-\frac{(\lambda-\mu)+\sqrt{(\lambda-\mu)^{2}+4 a}}{2 a}
$$

and conclude that

$$
\begin{aligned}
\frac{d \Phi}{d t} \leq & -\frac{1}{2}\left[(\lambda+\mu)-\left\{(\lambda-\mu)^{2}+4 a\right\}^{1 / 2}\right] \Phi \\
& +\left[\frac{27 \nu^{4}}{2}\left\{\int_{\Omega} v_{i, j} v_{i, j} d x\right\}^{2}+\frac{\nu^{4 / 3}}{2 a^{2}}\left\{\int_{\Omega} T^{6} d x\right\}^{2 / 3}\right] \Phi+\alpha^{2} \kappa^{-1} \int_{\Gamma}\left[T^{*}\right]^{2} d \sigma,
\end{aligned}
$$

where $\mu=\mu(\kappa, 1)$. We now choose the constant $a$ so that the quantity in square brackets in the first term on the right is positive. Any value of $a$ less than $\lambda \mu$ will suffice. We observe that as $a$ decreases the coefficient of $\left\{\int_{\Omega} T^{6} d x\right\}^{2 / 3}$ increases. We could, for instance, choose $a=\frac{\lambda \mu}{2}$, but in any case we are led to an inequality of the form

$$
\begin{aligned}
\frac{d \Phi}{d t} \leq- & A_{1} \Phi+A_{2}\left[\int_{\Omega} v_{i, j} v_{i, j} d x\right]^{2} \Phi \\
& +A_{3}\left[\int_{\Omega} T^{6} d x\right]^{2 / 3} \Phi+\alpha^{2} \kappa^{-1} \int_{\Gamma^{\top}}\left[T^{*}\right]^{2} d \sigma .
\end{aligned}
$$

We have already bounded some of the terms on the right, but we need a bound for $\int_{\Omega} v_{i, j} v_{i, j} d x$. With sufficient smoothness and compatibility, a bound for this expression 
is given by (A.22) in the Appendix, where it is shown that for sufficiently small data or on the interval $\left(0, \min \left\{t_{0}, t_{1}\right\}\right)$ (where the definition of $t_{1}$ is given by $(\mathrm{A} .20)$ )

$$
\int_{\Omega} v_{i, j} v_{i, j} d x \leq H_{2}(t) e^{-\frac{1}{2} \mu(\kappa, 1) t} .
$$

The insertion of (3.24) into (3.23) then leads to an inequality of the form

$$
\begin{aligned}
\frac{d \Phi}{d t} \leq- & A_{1} \Phi+A_{2} H_{2}^{2}(t) e^{-\mu(\kappa, 1) t} \Phi \\
& +A_{3} B_{10} e^{-\frac{20}{9} \mu(\kappa, 3) t} \Phi+\alpha^{2} \kappa^{-1} \int_{\Gamma}\left[T^{*}\right]^{2} d \sigma
\end{aligned}
$$

The expression for $H_{2}(t)$ is given by (A.23). For sufficiently small data, $Q_{1}(t)$ may be replaced by $Q_{1}(0)$ and (3.25) integrates to give

$$
\Phi(t) \leq M_{2} \alpha^{2} \int_{0}^{t} e^{-A_{1}(t-\eta)}\left\{\int_{\Gamma}\left[T^{*}\right]^{2} d \sigma\right\} d \eta
$$

Otherwise, for $0 \leq t<\min \left\{t_{0}, t_{1}\right\}$ we obtain an inequality of type (3.26) in which $M_{2}$ is now a function of $t$ that tends to infinity as $t \rightarrow t_{1}$. The continuous dependence inequality is finally made explicit by bounding the integral on the right of (3.26) in terms of data. This bound follows from (2.21) with $\beta=A_{1}$. Thus we obtain

$$
\Phi(t) \leq \frac{2 M_{2} \mu(\kappa, 1) \alpha^{2}}{\left[2 \mu(\kappa, 1)-A_{1}\right]} \int_{\Omega} T_{0}^{2} d x e^{-A_{1} t}
$$

is valid for $0 \leq t<\min \left\{t_{0}, t_{1}\right\}$, which establishes continuous dependence on the parameter $\kappa$.

We have established the following result:

THEOREM 1. Let $\left(v_{i}, T\right)$ and $\left(v_{i}^{*}, T^{*}\right)$ be solutions of $(1.1)-(1.3)$ subject to conditions (1.4) $-(1.5)$ and (3.1), (3.2) respectively. Then if $\Gamma$ is smooth, the data are compatible on $\Gamma \times\{0\}$ and $a$ lies in the interval $(0, \lambda \mu(\kappa, 1))$, it follows that for $t<\min \left\{t_{0}, t_{1}\right\}$ with $t_{1}$ defined by (A.20)

$$
\int_{\Omega}\left[\left(T-T^{*}\right)^{2}+a\left(v_{i}-v_{i}^{*}\right)\left(v_{i}-v_{i}^{*}\right)\right] d x \leq P\left(t, t_{1}, T_{0}, v^{0}\right)\left(\kappa-\kappa^{*}\right)^{2} e^{-A_{1} t} .
$$

Here $P\left(t, t_{1}, T_{0}, v^{0}\right)$ is a computable quantity that tends to infinity as $t \rightarrow t_{1}$, and

$$
A_{1}=\frac{1}{2}\left[(\lambda+\mu(\kappa, 1))-\left\{(\lambda-\mu(\kappa, 1))^{2}+4 a\right\}^{1 / 2}\right] .
$$

It is clear that this result is far from optimal because of the more or less arbitrary choices made for the constants arising in the derivation. Different choices for values of the constants could lead to a larger interval of continuous dependence. We have not pursued the question of the optimal choice for these constants. We should point out that (3.27) holds for $t_{0}$ in the indicated range as long as the base flow remains bounded on that interval. 
4. The forward-in-time problem in $\mathbb{R}^{2}$. The derivation of the stability inequality is much easier in $\mathbb{R}^{2}$ due to the use of the Sobolev inequality (2.23) instead of $(2.24)$. We proceed as in the three-dimensional case through (3.12), but instead of (3.13) we have in $\mathbb{R}^{2}$

$$
\begin{aligned}
& \left|\int_{\Omega} u_{i} \theta,_{i} T d x\right| \\
& \quad \leq\left[\int_{\Omega} \theta,_{i} \theta,_{i} d x\right]^{1 / 2}\left\{\int_{\Omega}\left[u_{i} u_{i}\right]^{2} d x\right\}^{1 / 4}\left\{\int_{\Omega} T^{4} d x\right\}^{1 / 4} \\
& \quad \leq \Lambda^{1 / 2}\left[\int_{\Omega} \theta,_{i} \theta,,_{i} d x\right]^{1 / 2}\left[\int_{\Omega} u_{i} u_{i} d x \int_{\Omega} u_{j, k} u_{j, k} d x \int_{\Omega} T^{4} d x\right]^{1 / 4} \\
& \quad \leq \frac{\Lambda^{1 / 2}}{2}\left\{\delta_{1}^{-1} \int_{\Omega} \theta,_{i} \theta,_{i} d x+\delta_{1}\left[\int_{\Omega} u_{i} u_{i} d x \int_{\Omega} u_{j, k} u_{j, k} d x \int_{\Omega} T^{4} d x\right]^{1 / 2}\right\} \\
& \quad \leq \frac{\Lambda^{1 / 2}}{2}\left\{\delta_{1}^{-1} \int_{\Omega} \theta,_{i} \theta,,_{i} d x+\frac{\delta_{1} \delta_{2}}{2} \int_{\Omega} u_{i, j} u_{i, j} d x+\frac{\delta_{1} \delta_{2}^{-1}}{2} \int_{\Omega} u_{i} u_{i} d x \int_{\Omega} T^{4} d x\right\} .
\end{aligned}
$$

Also in $\mathbb{R}^{2}$

$$
\begin{aligned}
\left|\int_{\Omega} u_{i} u_{j} v_{i, j} d x\right| & \leq\left[\int_{\Omega}\left[u_{i} u_{i}\right]^{2} d x \int_{\Omega} v_{i, j} v_{i, j} d x\right\}^{1 / 2} \\
& \leq \Lambda\left\{\int_{\Omega} u_{i} u_{i} d x \int_{\Omega} u_{j, k} u_{j, k} d x \int_{\Omega} v_{l, m} v_{l, m} d x\right\}^{1 / 2} \\
& \leq \frac{\Lambda \delta_{3}}{2} \int_{\Omega} u_{i} u_{i} d x \int_{\Omega} v_{l, m} v_{l, m} d x+\frac{\Lambda \delta_{3}^{-1}}{2} \int_{\Omega} u_{i, j} u_{i, j} d x
\end{aligned}
$$

Inequalities (3.16) and (3.17) are both valid in $\mathbb{R}^{2}$. Assembling terms we have

$$
\begin{aligned}
\frac{d \Phi}{d t} \leq- & \left(2-\Lambda^{1 / 2} \delta_{1}^{-1}\right) \int_{\Omega} \theta,_{i} \theta,,_{i}+\left(2 \kappa-\gamma_{4}^{-1}\right) \int_{\Gamma} \theta^{2} d \sigma+a \gamma_{5} \int_{\Omega} \theta^{2} d x+\alpha^{2} \gamma_{4} \int_{\Omega}\left[T^{*}\right]^{2} d \sigma \\
& -\left(2 a-\frac{\Lambda^{1 / 2} \delta_{1} \delta_{2}}{2} \Lambda \delta_{3}^{-1} a\right) \int_{\Omega} u_{i, j} u_{i, j} d x \\
+ & \left\{\gamma_{5}^{-1} a+\Lambda \delta_{3} a \int_{\Omega} v_{i, j} v_{i, j} d x+\frac{\Lambda^{1 / 2} \delta_{1}}{2} \delta_{2}^{-1} \int_{\Omega} T^{4} d x\right\} \int_{\Omega} u_{i} u_{i} d x .
\end{aligned}
$$

We now make the choices

$$
\delta_{1}=\Lambda^{1 / 2} ; \quad \delta_{2}=\Lambda^{-1} a ; \quad \delta_{3}=2 \Lambda ; \quad \gamma_{4}=\kappa^{-1}
$$

and are led to

$$
\begin{aligned}
\frac{d \Phi}{d t} \leq- & {\left[\left(\mu(\kappa, 1)-a \gamma_{5}\right] \int_{\Omega} \theta^{2} d x+\alpha^{2} \kappa^{-1} \int_{\Gamma}\left[T^{*}\right]^{2} d \sigma-a\left[\lambda-\gamma_{5}^{-1}\right] \int_{\Omega} u_{i} u_{i} d x\right.} \\
& +2 \Lambda^{2} a \int_{\Omega} v_{i, j} v_{i, j} d x \int_{\Omega} u_{k} u_{k} d x+\frac{\Lambda^{2}}{2 a} \int_{\Omega} T^{4} d x \int_{\Omega} u_{i} u_{i} d x .
\end{aligned}
$$


We again choose $\gamma_{5}$ as in (3.21) and find

$$
\begin{aligned}
\frac{d \Phi}{d t} \leq- & \frac{1}{2}\left[(\lambda+\mu)-\left\{(\lambda-\mu)^{2}+4 a\right\}^{1 / 2} \Phi\right. \\
& +\left[2 \Lambda^{2} \int_{\Omega} v_{i, j} v_{i, j} d x+\frac{\Lambda^{2}}{2 a^{2}} \int_{\Omega} T^{4} d x\right] \Phi+\alpha^{2} \kappa^{-1} \int_{\Gamma}\left(T^{*}\right)^{2} d \sigma .
\end{aligned}
$$

Again we write the inequality as

$$
\begin{aligned}
\frac{d \Phi}{d t} \leq- & \widetilde{A}_{1} \Phi+\widetilde{A}_{2}\left(\int_{\Omega} v_{i, j} v_{i, j} d x\right) \Phi \\
& +\widetilde{A}_{3}\left(\int_{\Omega} T^{4} d x\right) \Phi+\alpha^{2} \kappa^{-1} \int_{\Gamma}\left(T^{*}\right) d \sigma
\end{aligned}
$$

which integrates to give

$$
\begin{aligned}
\Phi(t) \leq \alpha^{2} \kappa^{-1}\left[\int _ { 0 } ^ { t } \operatorname { e x p } \left\{-\widetilde{A}_{1}(t-\eta)\right.\right. & +\widetilde{A}_{2} \int_{\eta}^{t} \int_{\Omega} v_{i, j} v_{i, j} d x d \rho \\
& \left.\left.+\widetilde{A}_{3} \int_{\eta}^{t} \int_{\Omega} T^{4} d x d \rho\right\} d \eta\right] \int_{\Gamma}\left(T^{*}\right)^{2} d \sigma .
\end{aligned}
$$

Integrating (2.17) from $\eta$ to $t$ and using (2.14) to bound $\int_{\Omega} v_{i} v_{i} d x$ at time $\eta$ we find

$$
\int_{\eta}^{t} \int_{\Omega} v_{i, j} v_{i, j} d x d \eta \leq\left(B_{6}+B_{7} \eta\right) e^{-\mu(\kappa, 1) \eta} .
$$

Also from (2.3)

$$
\int_{\eta}^{t} \int_{\Omega} T^{4} d x d \eta \leq\left[\int_{\Omega} T_{0}^{4} d x\right][3 \mu(\kappa, 2)]^{-1} e^{-3 \mu(\kappa, 2) \eta} .
$$

Inserting (4.8) and (4.9) into (4.7) we find

$$
\begin{aligned}
\Phi(t) \leq \alpha^{2} \kappa^{-1} \int_{0}^{t}\left[\operatorname { e x p } \left\{-\widetilde{A}_{1}(t-\eta)+\right.\right. & \widetilde{A}_{2}\left(B_{6}+B_{7} \eta\right) e^{-\mu(\kappa, 1) \eta} \\
& \left.\left.+\widetilde{A}_{3} B_{8} e^{-3 \mu(\kappa, 2) \eta}\right\} \int_{\Gamma}\left[T^{*}\right]^{2} d \sigma\right] d \eta \\
\leq & \alpha^{2} M_{3} \int_{0}^{t} \int_{\Gamma} e^{-\tilde{A}_{1}(t-\eta)}\left[T^{*}\right]^{2} d \sigma d \eta .
\end{aligned}
$$

The bound for the last integral is carried out just as in the derivation of the bound for the right-hand side of $(3.26)$ in $\mathbb{R}^{3}$. Thus we find

$$
\Phi(t) \leq\left\{\frac{2 M_{3} \mu(\kappa, 1)}{\left[2 \mu(\kappa, 1)-\widetilde{A}_{1}\right]}\right\} \alpha^{2}\left\{\int_{\Omega} T_{0}^{2} d x\right\} e^{-\widetilde{A}_{1} t} .
$$

We have proved

THEOREM 2. If $\left(v_{i}, T\right)$ and $\left(v_{i}^{*}, T^{*}\right)$ are two solutions of the system (1.1)-(1.3) with $\Omega \subseteq \mathbb{R}^{2}$ that satisfy the same auxiliary conditions except that (3.1) is satisfied by $T^{*}$, then for a constant $a$ and function $F(t)$ depending on the data,

$$
\int_{\Omega}\left[\left(T-T^{*}\right)^{2}+a\left(v_{i}-v_{i}^{*}\right)\left(v_{i}-v_{i}^{*}\right)\right] d x \leq\left(\kappa-\kappa^{*}\right)^{2} F(t) .
$$


It is interesting that in $\mathbb{R}^{2}$ our stability result has involved neither bounds for $\int_{\Omega} v_{i, j} v_{i, j} d x$ nor $\int_{\Omega} v_{i, t} v_{i, t} d x$. Consequently, it has not been necessary to assume that the differential equation is satisfied on $t=0$. We note further that in $\mathbb{R}^{2}$ we have not had to impose smallness assumptions on the initial data in order to obtain a stability inequality that is valid for all $t$. This means that in $\mathbb{R}^{2}$ we may permit $t_{0}$ to go to infinity. These results are similar to those obtained by Song [9] for the Navier-Stokes equations.

5. Backward-in-time problem. Instead of considering the original equations backward in time, we will replace $t$ by $-t$ and study the resulting system forward in time. The governing perturbation equations can then be written

$$
\begin{aligned}
& -u_{i, t}+v_{j}^{*} u_{i, j}+u_{j} v_{i, j}=-P,_{i}+\Delta u_{i}+b_{i} \theta \\
& u_{i, i}=0 \\
& \text { in } \Omega \times\left(0, t_{0}\right) \\
& -\theta,{ }_{t}+v_{i}^{*} \theta,_{i}+u_{i} T,_{i}=\Delta \theta
\end{aligned}
$$

with

$$
\begin{gathered}
u_{i}=0 \\
\theta_{. i} n_{i}+\kappa \theta=-\alpha T^{*} \text { on } \Gamma \times\left[0, t_{0}\right]
\end{gathered}
$$

and

$$
\theta(x, 0)=u_{i}(x, 0)=0, \quad x \in \Omega
$$

Here $u_{i}=v_{i}^{*}-v_{i}, \theta=T^{*}-T, P=p^{*}-p$, and $\alpha=\kappa^{*}-\kappa$. We assume, without loss, that

$$
\left|b_{i}\right| \leq 1
$$

Continuous dependence of solutions on the parameter $\alpha$ can be obtained using logarithmic convexity arguments (see Payne [6]). We thus introduce the functional

$$
\Phi(t)=\int_{0}^{t} \int_{\Omega}(t-\eta)^{2}\left(u_{i} u_{i}+\theta^{2}\right) d x d \eta+\alpha^{2} Q^{2}
$$

where $Q^{2}$ is a constant data term to be chosen. We now show that there exist constants $a_{1}$ and $a_{2}$ such that

$$
\Phi \Phi^{\prime \prime}-\left(\Phi^{\prime}\right)^{2} \geq-a_{1} \Phi \Phi^{\prime}-a_{2} \Phi^{2}
$$

To this end, we differentiate (5.8) with respect to $t$ to obtain

$$
\begin{aligned}
\Phi^{\prime}(t) & =2 \int_{0}^{t} \int_{\Omega}(t-\eta)\left(u_{i} u_{i}+\theta^{2}\right) d x d \eta \\
& =2 \int_{0}^{t} \int_{\Omega}(t-\eta)^{2}\left(u_{i} u_{i, \eta}+\theta \theta,_{\eta}\right) d x d \eta .
\end{aligned}
$$


Substitution of the differential equations (5.1), (5.2), and (5.3) and integration by parts results in

$$
\begin{aligned}
\Phi^{\prime}(t)=2 & \int_{0}^{t} \int_{\Omega}(t-\eta)^{2} u_{i} u_{j} v_{i, j} d x d \eta-2 \int_{0}^{t} \int_{\Omega}(t-\eta)^{2} b_{i} u_{i} \theta d x d \eta \\
& +2 \int_{0}^{t} \int_{\Omega}(t-\eta)^{2} u_{i, j} u_{i, j} d x d \eta+2 \int_{0}^{t} \int_{\Omega}(t-\eta)^{2} \theta u_{i} T,_{i} d x d \eta \\
& +2 \int_{0}^{t} \int_{\Omega}(t-\eta)^{2} \theta_{, i} \theta_{, i} d x d \eta+2 \int_{0}^{t} \int_{\Gamma}(t-\eta)^{2}\left(\kappa \theta^{2}+\alpha \theta T^{*}\right) d \sigma d \eta .
\end{aligned}
$$

Differentiating (5.12), we are led to

$$
\begin{aligned}
\Phi^{\prime \prime}(t)= & 4 \int_{0}^{t} \int_{\Omega}(t-\eta) u_{i} u_{j} v_{i, j} d x d \eta-4 \int_{0}^{t} \int_{\Omega}(t-\eta) b_{i} u_{i} \theta d x d \eta \\
& -4 \int_{0}^{t} \int_{\Omega}(t-\eta)^{2} u_{i, \eta} \Delta u_{i} d x d \eta+4 \int_{0}^{t} \int_{\Omega}(t-\eta) \theta u_{i} T,_{i} d x d \eta \\
& -4 \int_{0}^{t} \int_{\Omega}(t-\eta)^{2} \theta,_{\eta} \Delta \theta d x d \eta+4 \int_{0}^{t} \int_{\Gamma}(t-\eta)\left(\kappa \theta^{2}+\alpha \theta T^{*}\right) d \sigma d \eta
\end{aligned}
$$

A second use of the differential equations allows us to write (5.13) as

$$
\begin{aligned}
\Phi^{\prime \prime}(t)= & 4 \int_{0}^{t} \int_{\Omega}(t-\eta)^{2}\left(\psi_{i} \psi_{i}+\chi^{2}\right) d x d \eta+4 \int_{0}^{t} \int_{\Omega}(t-\eta) u_{i} u_{j} v_{i, j} d x d \eta \\
& -4 \int_{0}^{t} \int_{\Omega}(t-\eta) b_{i} u_{i} \theta d x d \eta-\int_{0}^{t} \int_{\Omega}(t-\eta)^{2} v_{j}^{*} u_{i, j} v_{l}^{*} u_{i, l} d x d \eta \\
& -4 \int_{0}^{t} \int_{\Omega}(t-\eta)^{2} u_{i, \eta} u_{j} v_{i, j} d x d \eta+4 \int_{0}^{t} \int_{\Omega}(t-\eta)^{2} u_{i, \eta} b_{i} \theta d x d \eta \\
& -\int_{0}^{t} \int_{\Omega}^{t}(t-\eta)^{2} v_{i}^{*} \theta,_{i} v_{l}^{*} \theta, l d x d \eta-4 \int_{0}^{t} \int_{\Omega}(t-\eta)^{2} u_{i} T,_{i} \theta,_{\eta} d x d \eta \\
& +4 \int_{0}^{t} \int_{\Omega}(t-\eta) \theta u_{i} T,_{i} d x d \eta+4 \int_{0}^{t} \int_{\Gamma}(t-\eta) \alpha \theta T^{*} d \sigma d \eta \\
& -4 \int_{0}^{t} \int_{\Gamma}(t-\eta)^{2} \alpha T^{*} \theta,_{\eta} d \sigma d \eta
\end{aligned}
$$

where

$$
\psi_{i}=u_{i, t}-\frac{1}{2} v_{j}^{*} u_{i, j}, \quad \chi=\theta_{t}-\frac{1}{2} v_{i}^{*} \theta,_{i}
$$

Observing that $\Phi^{\prime}$ can be written as

$$
\Phi^{\prime}=2 \int_{0}^{t} \int_{\Omega}(t-\eta)^{2}\left(u_{i} \psi_{i}+\theta \chi\right) d x d \eta
$$


we next form $\Phi \Phi^{\prime \prime}-\left(\Phi^{\prime}\right)^{2}$ to obtain

$$
\begin{aligned}
\Phi \Phi^{\prime \prime}-\left(\Phi^{\prime}\right)^{2} \geq & 4 S^{2}+4 \Phi \int_{0}^{t} \int_{\Omega}(t-\eta) u_{i} u_{j} v_{i, j} d x d \eta-4 \Phi \int_{0}^{t} \int_{\Omega}(t-\eta) b_{i} u_{i} \theta d x d \eta \\
& -\Phi \int_{0}^{t} \int_{\Omega}(t-\eta)^{2} v_{j}^{*} u_{i, j} v_{l}^{*} u_{i, l} d x d \eta-4 \Phi \int_{0}^{t} \int_{\Omega}(t-\eta)^{2} u_{i, \eta} u_{j} v_{i, j} d x d \eta \\
& +4 \Phi \int_{0}^{t} \int_{\Omega}(t-\eta)^{2} u_{i, \eta} b_{i} \theta d x d \eta-\Phi \int_{0}^{t} \int_{\Omega}(t-\eta)^{2} v_{i}^{*} \theta,_{i} v_{l}^{*} \theta,,_{l} d x d \eta \\
& -4 \Phi \int_{0}^{t} \int_{\Omega}(t-\eta)^{2} u_{i} T,_{i} \theta,_{\eta} d x d \eta+4 \Phi \int_{0}^{t} \int_{\Omega}(t-\eta) \theta u_{i} T,_{i} d x d \eta \\
& +4 \Phi\left[\int_{0}^{t} \int_{\Gamma}(t-\eta) \alpha \theta T^{*} d \sigma d \eta-\int_{0}^{t} \int_{\Gamma}(t-\eta)^{2} \alpha T^{*} \theta,_{\eta} d \sigma d \eta\right] \\
= & 4 S^{2}+\sum_{n=1}^{8} I_{n}+4 \Phi \int_{0}^{t} \int_{\Gamma}(t-\eta) \alpha \theta T^{*} d \sigma d \eta-\int_{0}^{t} \int_{\Gamma}(t-\eta)^{2} \alpha T^{*} \theta,_{\eta} d \sigma d \eta
\end{aligned}
$$

where

$$
\begin{aligned}
S^{2}= & {\left[\int_{0}^{t} \int_{\Omega}(t-\eta)^{2}\left(\psi_{i} \psi_{i}+\chi^{2}\right) d x d \eta\right]\left[\int_{0}^{t} \int_{\Omega}(t-\eta)^{2}\left(u_{i} u_{i}+\theta^{2}\right) d x d \eta\right] } \\
& \left.-\left(\int_{0}^{t} \int_{\Omega}(t-\eta)^{2}\left(u_{i} \psi_{i}+\theta \chi\right) d x d \eta\right)\right)^{2} .
\end{aligned}
$$

Clearly, $S^{2}$ is nonnegative by Schwarz's inequality. It also follows from (5.18) that

$$
\left[\int_{0}^{t} \int_{\Omega}(t-\eta)^{2}\left(\psi_{i} \psi_{i}+\chi^{2}\right) d x d \eta\right]^{1 / 2}\left[\int_{0}^{t} \int_{\Omega}(t-\eta)^{2}\left(u_{i} u_{i}+\theta^{2}\right) d x d \eta\right]^{1 / 2} \leq S+\frac{1}{2} \Phi^{\prime}
$$

We now proceed to bound each of the integrals on the right-hand side of (5.17) in terms of $\Phi^{2}$ and $\Phi \Phi^{\prime}$. In order to accomplish this, we assume that, uniformly in $\Omega \times\left[0, t_{0}\right)$,

$$
\left|v^{*}\right|,|\nabla v|,|\nabla T| \leq M
$$

for a positive constant $M$. Such an assumption restricts the class of solutions we shall consider in the sequel.

Rewriting (5.12) and applying Schwarz's inequality as well as (5.20), and the arithmetic-geometric mean inequality, we find that

$$
\begin{aligned}
& -\int_{0}^{t} \int_{\Omega}(t-\eta)^{2}\left(u_{i, j} u_{i, j}+\theta,{ }_{,} \theta,{ }_{i}\right) d x d \eta \\
& \quad \geq-\frac{1}{2} \Phi^{\prime}-\frac{1}{2}(3 M+1) \Phi+\int_{0}^{t} \int_{\Gamma}(t-\eta)^{2}\left[\kappa \theta^{2}+\alpha \theta T^{*}\right] d \sigma d \eta
\end{aligned}
$$


The following bounds also follow from (5.20) and the arithmetic-geometric mean inequality:

$$
\begin{aligned}
& I_{1} \geq-4 \Phi M \int_{0}^{t} \int_{\Omega}(t-\eta) u_{i} u_{i} d x d \eta \geq-2 M \Phi \Phi^{\prime}, \\
& I_{2} \geq-2 \Phi \int_{0}^{t} \int_{\Omega}(t-\eta)\left(u_{i} u_{i}+\theta^{2}\right) d x d \eta \geq-\Phi \Phi^{\prime}, \\
& I_{3} \geq-4 \Phi M^{2} \int_{0}^{t} \int_{\Omega}(t-\eta)^{2} u_{i, j} u_{i, j} d x d \eta, \\
& I_{6} \geq-4 \Phi M^{2} \int_{0}^{t} \int_{\Omega}(t-\eta)^{2} \theta,_{i} \theta,_{i} d x d \eta, \\
& I_{8} \geq-4 \Phi M \int_{0}^{t} \int_{\Omega}(t-\eta)\left(u_{i} u_{i}+\theta^{2}\right) d x d \eta \geq-2 M \Phi \Phi^{\prime} .
\end{aligned}
$$

To bound $I_{4}$, we observe that

$$
\begin{gathered}
-4 \Phi \int_{0}^{t} \int_{\Omega}(t-\eta)^{2} u_{i, \eta} u_{j} v_{i, j} d x d \eta=-4 \Phi \int_{0}^{t} \int_{\Omega}(t-\eta)^{2}\left(u_{i, \eta}-\frac{1}{2} v_{k}^{*} u_{i, k}\right) u_{j} v_{i, j} d x d \eta \\
-2 \Phi \int_{0}^{t} \int_{\Omega}(t-\eta)^{2} v_{k}^{*} u_{i, k} u_{j} v_{i, j}
\end{gathered}
$$

so that

$$
\begin{gathered}
I_{4} \geq-4 M \Phi\left(\int_{0}^{t} \int_{\Omega}(t-\eta)^{2} \psi_{i} \psi_{i} d x d \eta\right)^{1 / 2}\left(\int_{0}^{t} \int_{\Omega}(t-\eta)^{2} u_{i} u_{i} d x d \eta\right)^{1 / 2} \\
-M^{2} \Phi\left[\int_{0}^{t} \int_{\Omega}(t-\eta)^{2} u_{i, j} u_{i, j} d x d \eta+\int_{0}^{t} \int_{\Omega}(t-\eta)^{2} u_{i} u_{i} d x d \eta\right]
\end{gathered}
$$

after using (5.20) and applying the arithmetic-geometric mean and Schwarz's inequalities. Similarly, we can bound $I_{5}$ and $I_{7}$ to obtain

$$
\begin{aligned}
I_{5} \geq- & 4 \Phi\left(\int_{0}^{t} \int_{\Omega}(t-\eta)^{2} \psi_{i} \psi_{i} d x d \eta\right)^{1 / 2}\left(\int_{0}^{t} \int_{\Omega}(t-\eta)^{2} \theta^{2} d x d \eta\right)^{1 / 2} \\
- & M \Phi\left[\int_{0}^{t} \int_{\Omega}(t-\eta)^{2} \theta^{2} d x d \eta+\int_{0}^{t} \int_{\Omega}(t-\eta)^{2} u_{i, j} u_{i, j} d x d \eta\right]
\end{aligned}
$$

and

$$
\begin{aligned}
I_{7} \geq- & 4 \Phi M\left(\int_{0}^{t} \int_{\Omega}(t-\eta)^{2} \chi^{2} d x d \eta\right)^{1 / 2}\left(\int_{0}^{t} \int_{\Omega}(t-\eta)^{2} u_{i} u_{i} d x d \eta\right)^{1 / 2} \\
- & M^{2} \Phi\left[\int_{0}^{t} \int_{\Omega}(t-\eta)^{2} \theta,{ }_{i} \theta,{ }_{i} d x d \eta+\int_{0}^{t} \int_{\Omega}(t-\eta)^{2} u_{i} u_{i} d x d \eta\right] .
\end{aligned}
$$

Rewriting (5.12), we note it follows from (5.20) that

$$
\begin{aligned}
-\int_{0}^{t} \int_{\Omega}(t-\eta)^{2}\left(u_{i, j} u_{i, j}+\theta,_{i} \theta,_{i}\right) d x d \eta \geq & -\frac{1}{2} \Phi^{\prime}-\frac{1}{2}(3 M+1) \Phi \\
& +\int_{0}^{t} \int_{\Gamma}(t-\eta)^{2}\left(\kappa \theta^{2}+\alpha \theta T^{*}\right) d \sigma d \eta .
\end{aligned}
$$


Combining the bounds for $I_{n}$ and using (5.19) and (5.31), we derive

$$
\begin{aligned}
\Phi \Phi^{\prime \prime}-\left(\Phi^{\prime}\right)^{2} \geq 4 S^{2} & -4(2 M+1) \Phi S-C_{1} \Phi^{2}-C_{2} \Phi \Phi^{\prime} \\
& +4 \Phi\left[\int_{0}^{t} \int_{\Gamma}(t-\eta) \alpha \theta T^{*} d \sigma d \eta-\int_{0}^{t} \int_{\Gamma}(t-\eta)^{2} \alpha T^{*} \theta,_{\eta} d \sigma d \eta\right] \\
& +\left(5 M^{2}+M\right) \Phi \int_{0}^{t} \int_{\Gamma}(t-\eta)^{2}\left(\kappa \theta^{2}+\alpha \theta T^{*}\right) d \sigma d \eta
\end{aligned}
$$

where

$$
\begin{aligned}
& C_{1}=\frac{1}{2}\left(6 M^{2}+M\right)(3 M+1)+M(2 M+1), \\
& C_{2}=\frac{1}{2}\left(6 M^{2}+17 M+6\right) .
\end{aligned}
$$

We now complete the square on the first two terms on the right side of (5.32) and discard the nonnegative part to obtain

$$
\begin{gathered}
\Phi \Phi^{\prime \prime}-\left(\Phi^{\prime}\right)^{2} \geq \\
-C_{3} \Phi^{2}-C_{2} \Phi \Phi^{\prime}+4 \Phi\left[\int_{0}^{t} \int_{\Gamma}(t-\eta) \alpha \theta T^{*} d \sigma d \eta\right. \\
\left.-\int_{0}^{t} \int_{\Gamma}(t-\eta)^{2} \alpha T^{*} \theta,_{\eta} d \sigma d \eta\right] \\
+\left(5 M^{2}+M\right) \Phi \int_{0}^{t} \int_{\Gamma}(t-\eta)^{2}\left(\kappa \theta^{2}+\alpha \theta T^{*}\right) d \sigma d \eta
\end{gathered}
$$

with $C_{3}=C_{1}+(2 M+1)^{2}$.

It remains for us to estimate the boundary terms in (5.35). Integration and use of the Schwarz inequality lead to the bounds

$$
\begin{aligned}
J_{1}=4 \Phi & {\left[\int_{0}^{t} \int_{\Gamma}(t-\eta) \alpha \theta T^{*} d \sigma d \eta-\int_{0}^{t} \int_{\Gamma}(t-\eta)^{2} \alpha T^{*} \theta,_{\eta} d \sigma d \eta\right] } \\
\geq- & 4 \Phi \alpha\left(\int_{0}^{t} \int_{\Gamma}(t-\eta)^{2} \theta^{2} d \sigma d \eta\right)^{1 / 2}\left(\int_{0}^{t} \int_{\Gamma} T^{2} d \sigma d \eta\right)^{1 / 2} \\
& -4 \Phi \alpha\left(\int_{0}^{t} \int_{\Gamma}(t-\eta)^{2} \theta^{2} d \sigma d \eta\right)^{1 / 2}\left(\int_{0}^{t} \int_{\Gamma}(t-\eta)^{2} T,_{\eta}^{2} d \sigma d \eta\right)^{1 / 2}
\end{aligned}
$$

and

$$
\begin{aligned}
J_{2}= & \left(5 M^{2}+M\right) \Phi \int_{0}^{t} \int_{\Gamma}(t-\eta)^{2}\left(\kappa \theta^{2}+\alpha \theta T^{*}\right) d \sigma d \eta \\
\geq & \left(5 M^{2}+M\right) \Phi(\kappa+\alpha) \int_{0}^{t} \int_{\Gamma}(t-\eta)^{2} \theta^{2} d \sigma d \eta \\
& \quad-\left(5 M^{2}+M\right) \Phi \alpha\left(\int_{0}^{t} \int_{\Gamma}(t-\eta)^{2} \theta^{2} d \sigma d \eta\right)^{1 / 2}\left(\int_{0}^{t} \int_{\Gamma}(t-\eta)^{2} T^{2} d \sigma d \eta\right)^{1 / 2} .
\end{aligned}
$$

Let us now assume that

$$
\int_{0}^{t} \int_{\Gamma} T^{2} d \sigma d \eta+\int_{0}^{t} \int_{\Gamma}(t-\eta)^{2} T,_{\eta}^{2} d \sigma d \eta \leq M^{2}
$$


Hence,

$$
J_{1} \geq-8 \alpha \Phi M\left(\int_{0}^{t} \int_{\Gamma}(t-\eta)^{2} \theta^{2} d \sigma d \eta\right)^{1 / 2} \geq-4 \Phi \frac{\alpha^{2} M^{2}}{\mu_{1}}+\mu_{1} \int_{0}^{t} \int_{\Gamma}(t-\eta)^{2} \theta^{2} d \sigma d \eta
$$

for a positive constant $\mu_{1}$. Also, we have

$$
\begin{aligned}
J_{2} \geq & \left(5 M^{2}+M\right) \Phi(\kappa+\alpha) \int_{0}^{t} \int_{\Gamma}(t-\eta)^{2} \theta^{2} d \sigma d \eta \\
& -\phi\left[\frac{\alpha^{2} M^{4}(5 M+1)^{2}}{2 \mu_{2}}+\frac{\mu_{2}}{2} \int_{0}^{t} \int_{\Gamma}(t-\eta)^{2} \theta^{2} d \sigma d \eta\right]
\end{aligned}
$$

upon applying the arithmetic-geometric mean inequality. To complete our bounds on $J_{1}$ and $J_{2}$, we now need to find a bound for $\int_{0}^{t} \int_{\Gamma}(t-\eta)^{2} \theta^{2} d \sigma d \eta$. We do this by considering the identity

$$
0=\int_{0}^{t} \int_{\Omega}(t-\eta)^{2} \theta\left\{-\theta,,_{\eta}+v_{i}^{*} \theta,_{i}+u_{i} T,_{i}-\Delta \theta\right\} d x d \eta
$$

Integrating by parts using the boundary conditions (5.4)-(5.5) and rearranging the expression leads to the inequality

$$
\begin{gathered}
(\kappa+\alpha) \int_{0}^{t} \int_{\Gamma}(t-\eta)^{2} \theta^{2} d \sigma d \eta \leq-\alpha \int_{0}^{t} \int_{\Omega}(t-\eta)^{2} \theta T d \sigma d \eta+\int_{0}^{t} \int_{\Omega}(t-\eta)^{2} \theta^{2} d x d \eta \\
-\int_{0}^{t} \int_{\Omega}(t-\eta)^{2} \theta u_{i} T,_{i} d x d \eta
\end{gathered}
$$

If we apply Schwarz's inequality to (5.42) and use the assumption (5.20) as well as the arithmetic-geometric mean inequality, we obtain

$$
\begin{aligned}
(\kappa+ & \alpha) \int_{0}^{t} \int_{\Gamma}(t-\eta)^{2} \theta^{2} d \sigma d \eta \\
\leq & \frac{\gamma}{2} \int_{0}^{t} \int_{\Gamma}(t-\eta)^{2} \theta^{2} d \sigma d \eta+\frac{\alpha^{2}}{2 \gamma} \int_{0}^{t} \int_{\Gamma}(t-\eta)^{2} T^{2} d \sigma d \eta \\
& +\int_{0}^{t} \int_{\Omega}(t-\eta) \theta^{2} d x d \eta+\frac{M}{2} \int_{0}^{t} \int_{\Omega}(t-\eta)^{2}\left[\theta^{2}+u_{i} u_{i}\right] d x d \eta .
\end{aligned}
$$

Recalling the definition of $\Phi$, the expression (5.10), and the assumption (5.38) about the boundary data, we then conclude that

$$
\left(\kappa+\alpha-\frac{\gamma}{2}\right) \int_{0}^{t} \int_{\Gamma}(t-\eta)^{2} \theta^{2} d \sigma d \eta \leq \frac{1}{2 \gamma} \alpha^{2} M^{2}+\Phi^{\prime}+\frac{1}{2} M \Phi .
$$

Letting $\kappa+\alpha-\frac{\gamma}{2}=\mu$, we choose $\gamma$ so that $\mu>0$ and (5.43) is the desired estimate. Consequently, if we choose $\mu_{1}=1$ and $\mu_{2}=2 M(5 M+1)(\kappa+\alpha)$ and define

$$
Q^{2}=\left(\frac{1}{2 \gamma}+\frac{1}{\mu}\right) M^{2}+M^{3}(5 M+1),
$$


then

$$
\begin{aligned}
& J_{1} \geq-2(M+4) \Phi^{2}-4 \Phi \Phi^{\prime} \\
& J_{2} \geq-\frac{1}{\kappa^{*}} \Phi^{2} .
\end{aligned}
$$

It then follows from (5.35) that there are positive constants $a_{1}$ and $a_{2}$ so that inequality (5.9) is satisfied. The change of variable $\xi=e^{-\alpha_{1} t}$ transforms (5.9) into

$$
\frac{d^{2}}{d \xi^{2}}\left[\ln \Phi \xi^{-a_{2} / a_{1}^{2}}\right] \geq 0
$$

and hence it follows from Jensen's inequality that

$$
\Phi(t) \leq e^{\left(-a_{2} / a_{1}\right) t}[\Phi(0)]^{\delta(t)}\left[\Phi\left(t_{0}\right) e^{\left(a_{2} t_{0} / a_{1}\right)}\right]^{1-\delta(t)}
$$

where

$$
0<\delta(t)=\frac{e^{-a_{1} t}-e^{-a_{1} t_{0}}}{1-e^{-a_{1} t_{0}}}<1
$$

Since

$$
\Phi(0)=\alpha^{2} Q^{2}
$$

and

$$
\Phi\left(t_{0}\right)=\int_{0}^{t_{0}} \int_{\Omega}\left(t_{0}-\eta\right)^{2}\left(u_{i} u_{i}+\theta^{2}\right) d x d \eta+\alpha^{2} Q^{2}
$$

inequality (5.47) will be the desired continuous dependence result provided we assume

$$
\Phi\left(t_{0}\right) e^{a_{2} t_{0} / a_{1}} \leq K^{2} .
$$

We thus conclude from (5.47) that

$$
\Phi(t) \leq C K^{2[1-\delta(t)]} \alpha^{2 \delta(t)}
$$

for $t \in\left[0, t_{0}\right)$ and a computable constant $C$. Hence solutions belonging to the appropriate class of functions depend Hölder continuously on the parameter $\alpha$ in the measure $\Phi$.

In summary, we have established the following result.

ThEOREM 3. Let $\left(v_{i}, T\right)$ and $\left(v_{i}^{*}, T^{*}\right)$ be two solutions of the system (1.1)-(1.3) backward in time that satisfy the same initial conditions (1.5) and boundary conditions on $v_{i}$ but have different parameters $\kappa$ and $\kappa^{*}$ in condition (1.4). If $v^{*}, v$, and $T$ belong to the classes of functions satisfying

$$
\left|v^{*}\right|,|\nabla v|,|\nabla T| \leq M
$$

and

$$
\int_{0}^{t} \int_{\Gamma} T^{2} d \sigma d \eta+\int_{0}^{t} \int_{\Gamma}(t-\eta) T_{\eta}^{2} d \sigma d \eta \leq M^{2}
$$

as well as the bound (5.49), then there exists a computable constant $B$ such that

$$
\int_{0}^{t} \int_{\Omega}(t-\eta)^{2}\left[\left(v_{i}-v_{i}^{*}\right)\left(v_{i}-v_{i}^{*}\right)+\left(T-T^{*}\right)^{2}\right] d x d \eta \leq B\left(\kappa-\kappa^{*}\right)^{\delta(t)}
$$

for $0<\delta(t)<1, t \in\left[0, t_{0}\right)$. 
Appendix. Our task here is to establish the inequality (3.24), but before starting the proof of this result let us first derive the following bound for the $L_{2}$ integral of $T_{,}$, which will be needed in the derivation of (3.24):

$$
\int_{\Omega(t)} T_{, t}^{2} d x \leq \int_{\Omega(0)} T_{, t}^{2} d x e^{-\mu(\kappa, 1) t}+\left|T_{0}\right|_{m}^{2} \int_{0}^{t} \int_{\Omega(\eta)} e^{-\mu(2 \kappa, 1)(t-\eta)} v_{i, t} v_{i, t} d x d \eta,
$$

where the symbol $\Omega(t)$ is used to indicate that integration is taken over $\Omega$ at time $t$, and $\left|T_{0}\right|_{m}$ is the maximum value of $\left|T_{0}\right|$ in $\Omega$.

To establish (A.1) we observe that

$$
\begin{aligned}
\frac{d}{d t} \int_{\Omega} T_{, t}^{2} d x= & 2 \int_{\Omega} T,_{t}\left\{\Delta T-T,_{i} v_{i}\right\}_{, t} d x \\
= & -2 \kappa \int_{\Gamma} T_{,_{t}}^{2} d \sigma-2 \int_{\Omega} T,_{i t} T,_{i t} d x \\
& \quad+2\left|T_{0}\right|_{m}\left[\int_{\Omega} T,_{i t} T,_{i t} d x \int_{\Omega} v_{i, t} v_{i, t} d x\right]^{1 / 2}
\end{aligned}
$$

Thus

$$
\frac{d}{d t} \int_{\Omega} T,_{t}^{2} d x \leq-2 \kappa \int_{\Gamma} T_{, t}^{2} d \sigma-\left(2-\gamma_{9}\right) \int_{\Omega} T_{, i t} T,{ }_{i t} d x+\gamma_{9}^{-1}\left|T_{0}\right|_{m}^{2} \int_{\Omega} v_{i, t} v,{ }_{i, t} d x .
$$

Choosing $\gamma_{9}=1$ we have

$$
\begin{aligned}
\frac{d}{d t} \int_{\Omega} T_{, t}^{2} d x & \leq-2 \kappa \int_{\Gamma} T_{, t}^{2} d \sigma-\int_{\Omega} T,{ }_{i t} T,{ }_{i t} d x+\left|T_{0}\right|_{m}^{2} \int_{\Omega} v_{i, t} v_{i, t} d x \\
& \leq-\mu(2 \kappa, 1) \int_{\Omega} T,_{t}^{2} d x+\left|T_{0}\right|_{m}^{2} \int_{\Omega} v_{i, t} v_{i, t} d x
\end{aligned}
$$

An integration of (A.4) leads directly to (A.1).

In general, the first integral term on the right of (A.1) is not data. However, if $\Gamma$ is sufficiently smooth and the data are compatible on the boundary $\Gamma$ at $t=0$ so that the $T$ equation is satisfied on $t=0$, we have

$$
\int_{\Omega(0)} T_{, t}^{2} d x=\int_{\Omega}\left[\Delta T_{0}-v_{i}^{0} T_{0, i}\right]^{2} d x
$$

which is a computable data term.

We are now ready to prove (3.24). We first note that

$$
\begin{aligned}
\int_{\Omega} v_{i, j} v_{i, j} d x & =-\int_{\Omega} v_{i}\left\{v_{i, t}+v_{j} v_{i, j}+p_{, i}-b_{i} T\right\} d x \\
& =-\int_{\Omega} v_{i} v_{i, t} d x+\int_{\Omega} b_{i} v_{i} T d x \\
& \leq\left[\int_{\Omega} v_{i} v_{i} d x\right]^{1 / 2}\left\{\left[\int_{\Omega} v_{i, t} v_{i, t} d x\right]^{1 / 2}+\left[\int_{\Omega} T^{2} d x\right]^{1 / 2}\right\} .
\end{aligned}
$$

A bound for $\int_{\Omega} v_{i} v_{i} d x$ is given by (2.16) and a bound for $\int_{\Omega} T^{2} d x$ by (2.3). It remains to bound the term $\int_{\Omega} v_{i, t} v_{i, t} d x$. 
Now

$$
\begin{aligned}
\frac{d}{d t} \int_{\Omega} v_{i, t} v_{i, t} d x= & 2 \int_{\Omega} v_{i, t}\left\{\Delta v_{i}-p,_{i}+b_{i} T-v_{j} v_{i, j}\right\}_{, t} d x \\
= & -2 \int_{\Omega} v_{i, j t} v_{i, j t} d x+2 \int_{\Omega} b_{i} v_{i, t} T d x-2 \int_{\Omega} v_{i, t} v_{j, t} v_{i, j} d x \\
\leq & -2 \int_{\Omega} v_{i, j t} v_{i, j t} d x+2\left[\int_{\Omega} T^{2} d x \int_{\Omega} v_{i, t} v_{i, t} d x\right]^{1 / 2} \\
& +2\left\{\int_{\Omega} v_{i, j} v_{i, j} d x \int_{\Omega}\left[v_{k, t} v_{k, t}\right]^{2} d x\right\}^{1 / 2} \\
\leq & -2 \int_{\Omega} v_{i, j t} v_{i, j t} d x+2\left[\int_{\Omega} T^{2} d x \int_{\Omega} v_{i, t} v_{i, t} d x\right]^{1 / 2} \\
& +2 \nu\left\{\left[\int_{\Omega} v_{i, j} v_{i, j} d x\right]_{\Omega}^{2} \int_{k, t} v_{k, t} d x\right\}^{1 / 4}\left\{\int_{\Omega} v_{i, j t} v_{i, j t} d x\right\}^{3 / 4} .
\end{aligned}
$$

Using the arithmetic-geometric mean and Young inequalities we have

$$
\begin{aligned}
\frac{d}{d t} \int_{\Omega} v_{i, t} v_{i, t} d x \leq & -\left(2-\frac{3}{2} \nu \gamma_{6}\right) \int_{\Omega} v_{i, j t} v_{i, j t} d x \\
& +\gamma_{7} \int_{\Omega} v_{i, t} v_{i, t} d x+\gamma_{7}^{-1} \int_{\Omega} T,_{t}^{2} d x \\
& +\frac{\nu}{2} \gamma_{6}^{-1}\left[\int_{\Omega} v_{i, j} v_{i, j} d x\right]^{2}\left[\int_{\Omega} v_{i, t} v_{i, t} d x\right] .
\end{aligned}
$$

We introduce the notation

$$
R(t)=\int_{\Omega} v_{i, t} v_{i, t} d x
$$

and make use of (A.6) to obtain for $\frac{3}{2} \nu \gamma_{6}<2$

$$
\begin{aligned}
\frac{d R}{d t} \leq\{ & \left.\gamma_{7}-\left(2-\frac{3}{2} \nu \gamma_{6}\right) \lambda\right\} R+\gamma_{7}^{-1} \int_{\Omega} T,{ }_{t}^{2} d x \\
& +\frac{\nu}{2} \gamma_{6}^{-1}\left[\int_{\Omega} v_{i} v_{i} d x\right]\left\{R^{1 / 2}+\left[\int_{\Omega} T^{2} d x\right]^{1 / 2}\right\}^{2} R \\
\leq- & \left\{2 \lambda-\frac{3}{2} \gamma_{6} \nu \lambda-\gamma_{7}\right\} R+\gamma_{7}^{-1} \int_{\Omega} T,{ }_{t}^{2} d x \\
& +\frac{\nu}{2} \gamma_{6}^{-1}\left[\int_{\Omega} v_{i} v_{i} d x\right]\left\{\left(1+\gamma_{8}\right) R^{2}+\gamma_{8}^{-1}\left(1+\gamma_{8}\right) \int_{\Omega} T^{2} d x R\right\} .
\end{aligned}
$$

We have used (2.11) and the fact that $v_{i, t}$ vanishes on $\Gamma$. Choosing

$$
\gamma_{6}=2[3 \nu]^{-1}, \quad \gamma_{7}=\lambda-\frac{1}{2} \mu(\kappa, 1),
$$


we have, using (A.1),

$$
\begin{aligned}
\frac{d R}{d t} \leq- & \frac{1}{2} \mu(\kappa, 1) R+\left[\lambda-\frac{1}{2} \mu(\kappa, 1)\right]^{-1}\left\{B_{1} e^{-\mu(\kappa, 1) t}+\left|T_{0}\right|_{m}^{2} \int_{0}^{t} e^{-\mu(2 \kappa, 1)(t-\eta)} R(\eta) d \eta\right\} \\
& +\left(B_{2}+B_{3} t\right) e^{-\mu(\kappa, 1) t} R^{2}+\left(B_{4}+B_{5} t\right) e^{-2 \mu(\kappa, 1) t} R,
\end{aligned}
$$

where the constants $B_{i}$ are computable data terms obtained from (2.3), (2.16), and (A.5).

We consider first the special case

$$
\left|T_{0}\right|_{\max }^{2} \leq \frac{1}{2} \mu(\kappa, 1) \mu(2 \kappa, 1)\left[\lambda-\frac{1}{2} \mu(\kappa, 1)\right] .
$$

In this case we have, setting

$$
S(t)=R(t)+\frac{1}{2} \mu(\kappa, 1) \int_{0}^{t} e^{-\mu(2 \kappa, 1)(t-\eta)} R(\eta) d \eta+Q_{0},
$$

where $Q_{0}$ is some sufficiently large data term,

$$
\frac{d S}{d t} \leq\left(B_{2}+B_{3} t\right) e^{-\mu(\kappa, 1) t} S^{2} .
$$

This integrates to give

$$
\begin{aligned}
\frac{1}{S(0)} & \leq \frac{1}{S(t)}+\int_{0}^{t}\left(B_{2}+B_{3} \eta\right) e^{-\mu(\kappa, 1) \eta} d \eta \\
& =\frac{1}{S(t)}+[\mu(\kappa, 1)]^{-2}\left\{\mu(\kappa, 1) B_{2}\left(1-e^{-\mu(\kappa, 1) t}\right)+B_{3}\left(1-e^{-\mu(\kappa, 1) t}\right.\right. \\
& \left.\left.=\frac{1}{S(t)} Q_{1}(t) . \mu(\kappa, 1) t e^{-\mu(\kappa, 1) t}\right)\right\}
\end{aligned}
$$

Thus, provided $Q_{1}(t)<[S(0)]^{-1}$, we have

$$
S(t) \leq\left[\frac{1}{S(0)}-Q_{1}(t)\right]^{-1} .
$$

Clearly, if

$$
\mu(\kappa, 1) B_{2}+B_{3}<[\mu(\kappa, 1)]^{2}[S(0)]^{-1},
$$

i.e., if

$$
\left[\mu(\kappa, 1) B_{2}+B_{3}\right]\left[R(0)+Q_{0}\right]<\mu(\kappa, 1)^{2}
$$

then (A.17) will hold for all time. This means that for sufficiently small data (A.17) will hold for all $t>0$ (i.e., $t_{0}$ can be taken arbitrarily large). If (A.19) does not hold then (A.17) is valid only for some finite time interval, i.e., for $t<t_{1}$, where

$$
Q_{1}\left(t_{1}\right) S(0)=1 \text {. }
$$

If (A.13) is satisfied then for any fixed time interval $\left(0, t_{0}\right)$ for finite $t_{0}$ we may choose $Q_{0}$ so large that (A.15) is satisfied. However, when (A.13) does not hold, $Q_{0}$ will behave like $\left(B_{2}+B_{3} t_{0}\right)^{-1} e^{\mu(\kappa, 1) t_{0}}$ and hence will go to infinity as $t_{0} \rightarrow \infty$. For fixed $t_{0}$ then (A.17) holds provided $Q_{1}(t)<[S(0)]^{-1}$. Recalling that $t_{1}$ is the value of $t$ at which $Q_{1}(t)=[S(0)]^{-1}$ then (A.17) holds for $0 \leq t<\min \left\{t_{0}, t_{1}\right\}$. 
Returning now to (A.6) we have

$$
\int_{\Omega} v_{i, j} v_{i, j} d x \leq\left\{\left(B_{6}+B_{7} t\right) R(t)\right\}^{1 / 2} e^{-\frac{1}{2} \mu(\kappa, 1) t}+\left(B_{8}+B_{9} t\right)^{1 / 2} e^{-\frac{3}{2} \mu(\kappa, 1) t} .
$$

Thus for sufficiently small data or on the interval $\left(0, \min \left\{t_{0}, t_{1}\right\}\right)$

$$
\begin{aligned}
\int_{\Omega} v_{i, j} v_{i, j} d x & \leq\left(B_{6}+B_{7} t\right)^{1 / 2}\left[\frac{1}{S(0)}-Q_{1}(t)\right]^{-1 / 2} e^{-\frac{1}{2} \mu(\kappa, 1) t}+\left(B_{8}+B_{9} t\right)^{1 / 2} e^{-\frac{3}{2} \mu(\kappa, 1) t} \\
& \leq H_{2}(t) e^{-\frac{1}{2} \mu(\kappa, 1) t}
\end{aligned}
$$

where

$$
H_{2}(t)=\left[B_{6}+B_{7} t\right]^{1 / 2}\left[\frac{1}{S(0)}-Q_{1}(t)\right]^{-1 / 2}+\left(B_{8}+B_{9} t\right)^{1 / 2} e^{-\mu(\kappa, 1) t}
$$

This establishes (3.24).

\section{REFERENCES}

[1] R. A. Adams, Sobolev Spaces, Academic Press, New York, 1975

[2] K. A. Ames and L. E. Payne, Continuous dependence results for solutions of the Navier-Stokes equations backward in time, Nonlinear Analysis, Theory, Methods and Appl. 23, 103-113 (1994)

[3] C. Bandle, Isoperimetric Inequalities and Applications, Pitman, Boston, 1980

[4] F. Franchi and B. Straughan, Continuous dependence on the body force for solutions to the NavierStokes equations and on the heat supply in a model for double diffusive porous convection, J. Math. Anal. Appl. 172, 117-129 (1993)

[5] R. J. Knops and L. E. Payne, On the stability of the Navier-Stokes equations backward in time, Arch. Rational Mech. Anal. 29, 331-335 (1968)

[6] L. E. Payne, Improperly Posed Problems in Partial Differential Equations, Regional Conference Series in Applied Mathematics, Vol. 22, SIAM, Philadelphia, 1975

[7] L. E. Payne and H. Weinberger, Lower bounds for vibration frequencies of elastically supported membranes and plates, J. Soc. Indust. Appl. Math. 5, 171-182 (1957)

[8] J. Serrin, The initial value problem for the Navier-Stokes equations, Proc. Sympos. Nonlinear Problems, University of Wisconsin Press, Madison, WI, pp. 69-98.

[9] J. C. Song, Continuous dependence on the initial data, viscosity, and body force for the NavierStokes equations, SAACM 2, 265-279 (1993)

[10] R. Sperb, Untere und obere Schranken für den tiefsten Eigenwert der elastisch gestützen Membran, ZAMP 23, 231-244 (1972)

[11] B. Straughan, Mathematical aspects of penetrative convection, Pitman Res. Notes Math., Vol. 288, Longman, Harlow, 1993.

[12] W. Velte, Über ein Stabilitätskriterium in Hydrodynamik, Arch. Rational Mech. Anal. 9, 9-20 (1962) 